\section{Bird species diversity}

SIR - Graham Martin's report ${ }^{1}$ of the recent symposium on avian systematics (Avian Taxonomy from Linnaeus to DNA; London, 23 March 1996) covered several important aspects of the debate over two different species concepts - the biological and phylogenetic - and how use of the latter might increase the number of bird species recognized. But he gave a somewhat partial view, and ornithologists do not need to "start saving for the double-sized revised editions".

In particular, Martin suggested that new methods of molecular analysis, such as geographical studies of mitochondrial DNA (mtDNA), would lead to a doubling of the currently recognized species diversity of birds. The figure Martin quoted of 20,000 species of birds, rather than the currently recognized $\sim 9,000$, did indeed come from my presentation, based in part on unpublished work by G. F. Barrowclough, J. Cracraft and myself. However, contrary to Martin's implication, that study involved neither molecular data nor minute morphological differences. It was based on re-evaluation of already known, broad patterns of morphological variation (as I indicated in my talk). Hence, the data to re-evaluate bird species diversity under alternative species concepts, such as a phylogenetic species concept ${ }^{2}$, already exist. Many field guides already figure the distinctive subspecies that would probably be considered species under the phylogenetic species concept, and many bird watchers can already distinguish these forms. Thus, field guides would not double in size.

Two other points merit mention. First, it is likely that the number of phylogenetic species in the world will be much less than the number of already recognized subspecies - so the phylogenetic species concept will probably not greatly increase the number of names needed. Indeed, probably only $50 \%$ of recognized subspecies would qualify as phylogenetic species (our unpublished data, mentioned above). Second, there are very few studies in which several genes provide data for subdivisions of species that are not already apparent from morphological studies. One study that Martin cites makes this clear $^{3}$. The bird pictured in his meeting report, the three-toed woodpecker (Picoides tridactyl$u s$ ), was the only species of 13 tested which showed major mtDNA distinctness without well-marked phenotypic variation (although the samples compared with

\footnotetext{
. Martin, G. Nature 380, 666-667 (1996).

. Cracraft, J. Curr. Ornith. 1, 159-187 (1983)

3. Zink, R. M. Evolution 48, 96-111 (1994).

. Zink, R. M. et al. Condor 97, 639-649 (1995).

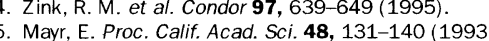

6. Graybeal, A. Syst. Biol. 44, 237-250 (1995).

Davis, J. I. Syst. Bot. 20, 555-559 (1995).
}

mtDNA were from different, previously recognized subspecies $)^{4}$. This hardly foreshadows a massive increase in species from molecular systematic studies. Thus, amateur and professional fieldworkers alike will not need DNA labs for field identification.

Molecular analyses are giving a refined assessment of patterns of variation within currently recognized biological species of birds. It is possible that there will be a 'class' of species defined by DNA markers which are difficult to distinguish in the field, but current evidence suggests this will not be an especially large category. There is indeed a serious debate about species

\title{
Skin grafts and cheetahs
}

SIR - Since the publication of the landmark study by O'Brien et al. ${ }^{1}$ on the lack of genetic variation in cheetahs, a flurry of reports have questioned this work ${ }^{2-6}$. This scepticism is due, in part, to the acceptance of reciprocal skin grafts between unrelated cheetahs reported by O'Brien et al., a phenomenon not previously observed in wild mammals. Such allogeneic graft acceptance suggests unusual monomorphism at the major histocompatibility complex (MHC), a group of loci responsible for many immune functions. These skin-graft results, a cornerstone of O'Brien et al.'s hypothesis on genetic vulnerability, have been criticized for problematic methodology and inconsistencies ${ }^{3}$. Recently, in Nature, $\mathrm{May}^{6}$ and Laurenson et al. ${ }^{7}$ characterized these trials as "dodgy" and "suspect", respectively, citing concerns first raised by Caughley ${ }^{3}$. Surprisingly, no attempts have been made to repeat this test on other wild species ${ }^{3}$.

We performed skin-graft experiments in Thomomys bottae, the pocket gopher. The populations of this species together span the range of mean heterozygosities $(H)$ found in all mammals ( $H=$ $0.01-0.24)^{8}$. We trapped individuals from two low-variation populations (Patricks J and Patricks $\mathrm{F}, H \approx 0.02$ ) and one highvariation population (Hastings, $H \approx 0.16$ ), and performed reciprocal skin grafts on within-population pairs of animals. Each animal received one allograft in addition to an autograft as a control. We monitored grafts for 3 months for signs of rejection. Accepted allografts became indistinguishable from autografts typically within 2 weeks after surgery. The few technical failures caused through graft injury were excluded from our analyses.

Multi-locus DNA fingerprinting analyses were performed on all individuals and band sharing was assessed ${ }^{9}$. Hastings animals showed low band sharing $(0.55$, $n=16$ ), whereas the Patricks $\mathrm{J}$ and Patricks F animals showed extreme sharing of bands within populations (0.97, $n=21 ; 0.98, n=7$, respectively) but low band sharing between populations (0.31). concepts, involving whether to give primary emphasis to the process of mate choice ${ }^{5}$ or evolutionary patterns ${ }^{6,7}$, corresponding to the biological species concept and a phylogenetic species concept. Although more species of birds are likely to be recognized with both molecular data and the phylogenetic species concept, it will not make field identification much harder than it is at present, and will lead to an improved understanding of the biology of birds.

Robert M. Zink

Bell Museum,

University of Minnesota,

St Paul, Minnesota 55108, USA
We used a subset of these animals for skin grafting. Essentially all Patricks $\mathbf{J}(n=14)$ and Patricks $\mathrm{F}(n=6)$ individuals accepted within-population allografts, whereas Hastings animals $(n=12)$ rejected all such grafts. The difference in acceptance between high- and low-variation populations was highly significant $\left(\chi^{2}=30\right.$, $P<0.0001)$. To test immunocompetence in the low-variation populations, we gave allografts from Hastings animals to each of three Patricks $J$ and two Patricks F animals that accepted within-population allografts; these grafts were rejected.

Skin graft acceptance is an indicator of genetic near-identity, at least at the MHC. Our results indicate that individuals from populations with low levels of genetic variation can have similar MHC genotypes, and we believe that the earlier cheetah results ${ }^{1}$, because of their concordance with our work, were indeed real phenomena. A consequence of such extreme homozygosity could be a loss of fitness caused by a reduction in immune-system pliancy ${ }^{1,10}$. Patricks $\mathbf{J}$ and Patricks F gophers, like many endangered species, appear to be highly homozygous, but are not immunocompromised and manage to persist in the wild. Such populations, however, assuming a concordance between MHC variability and disease susceptibility, may be equally vulnerable (or resistant) to particular pathogens.

\section{A. Sanjayan, Kevin Crooks}

Department of Biology,

Sinsheimer Laboratories,

University of California,

Santa Cruz, California 95064, USA

1. O'Brien, S. J. et al. Science 227, 1428-1434 (1985).

. Caro, T. M. \& Laurenson, M. K. Science 263, 485-486 1994)

Caughley, G. J. Anim. Ecol. 63, 215-244 (1994).

. Merola, M. Conserv. Biol. 8, 961-971 (1994)

5. O'Brien, S. J. Conserv. Biol. 8, 1153-1155 (1994).

6. May, R. M. Nature 374, 309-310 (1995)

7. Laurenson, M. K. et al. Nature 377, 392 (1995).

8. Patton, J. L. \& Smith, M. F. The Evolutionary Dynamics of the Pocket Gopher Thomomys Bottae, with Emphasis on California Populations (Univ. California Press, Berkeley, 1990).

9. Zimmerman, R. C. SIM 1.1 Prog. (Univ. California, Los Angeles, 1993)

10. Thorne, T. E. \& Williams, E. S. Conserv. Biol. 2, 66-74 (1988) 